\title{
Hiding in the dark: pan-cancer characterization of expression and clinical relevance of CD40 to immune checkpoint blockade therapy
}

\author{
Chi Yan ${ }^{1,2}$ and Ann Richmond ${ }^{1,2^{*}}$
}

Highlights: - $C D 40$ expression correlates with the type I anti-tumor response and better survival.

- Pan-cancer bioinformatics characterization reveals reduced CD40 expression in 11 cancer types, including RAS${ }^{m u t}$ melanoma compared to nevi.

- $R A S$ mutation correlates with reduced CD40 expression in malignant melanoma.

- $C D 40$ expression is associated with better response to immune checkpoint blockade therapy in melanoma.

Keywords: CD40, Cancer, Nevi, Melanoma, PD-1/PD-L1, CTLA4, Targeted therapy, Immune therapy

\begin{abstract}
Main text
CD40, also known as TNFRSF5, is a co-stimulatory cell surface receptor present on antigen-presenting cells (APC), non-hematopoietic cells (e.g., myofibroblasts, fibroblasts, epithelial, and endothelial cells) and tumor cells $[1,2]$. The CD40 and CD40 ligand (CD40LG) interaction mediates anti-tumoral immune responses by increasing immunogenic cell death (ICD) of tumor cells, APC activation, tumor immunogenicity by up-regulating major histocompatibility complex (MHC) molecules, production of proinflammatory factors, costimulation of $\mathrm{CD}^{+}$and $\mathrm{CD} 8^{+} \mathrm{T}$ cells, and tumor cell susceptibility to T-cell lysis [2-4]. Recently, our group demonstrated that RAS/RAF/PI3K pathway inhibition promotes ICD and augments response to immune checkpoint blockade (ICB), which mechanistically relies on CD40 induction by melanoma cells [2]. While the prevalence and importance of RAS/RAF/PI3K activating mutations across a very
\end{abstract}

*Correspondence: ann.richmond@vanderbilt.edu

2 Department of Pharmacology, Vanderbilt University School of Medicine, 432 PRB, 2220 Pierce Ave, Nashville, TN 37232, USA

Full list of author information is available at the end of the article broad spectrum of human cancer types is clear, we still lack understanding of whether CD40 regulation by the RAS/RAF/PI3K pathway broadly serves as an immune evasion/tolerance mechanism in tumor progression and response to therapy. In this study, we systematically characterized the expression and clinical relevance of CD40 across a variety of normal tissues and cancer types.

\section{CD40 expression correlates with the type-I anti-tumor response and better clinical outcome} Using the TCGA/TARGET/GTEx RNA-Seq dataset which covers 23 normal tissues and corresponding cancer types (Fig. 1A), we analyzed CD40 mRNA expression and its association to its ligand (CD4OLG), markers of MHC (HLA-DRA), dendritic cells (BATF3), $\mathrm{B}$ cells $(P A X 5)$, macrophages (CD68), granulocytes (mainly neutrophils, CD177), T-cells (CD4 and CD8A), type-I anti-tumor responses (IFNG and GZMB), cell proliferation (MKI67), cell death $(B A X)$, cell survival (BCL2) and type-II pro-tumor responses (IL5) in normal $(n=8152)$ and cancer $(n=10,137)$ tissues (Fig. 1B). Using Spearmen $r \geq 0.3$ or $r \leq-0.3$ and $p<0.001$ as a cut-off, we showed that $C D 40$ is original author(s) and the source, provide a link to the Creative Commons licence, and indicate if changes were made. The images or other third party material in this article are included in the article's Creative Commons licence, unless indicated otherwise in a credit line to the material. If material is not included in the article's Creative Commons licence and your intended use is not permitted by statutory regulation or exceeds the permitted use, you will need to obtain permission directly from the copyright holder. To view a copy of this licence, visit http://creativecommons.org/licenses/by/4.0/. The Creative Commons Public Domain Dedication waiver (http://creativeco mmons.org/publicdomain/zero/1.0/) applies to the data made available in this article, unless otherwise stated in a credit line to the data. 


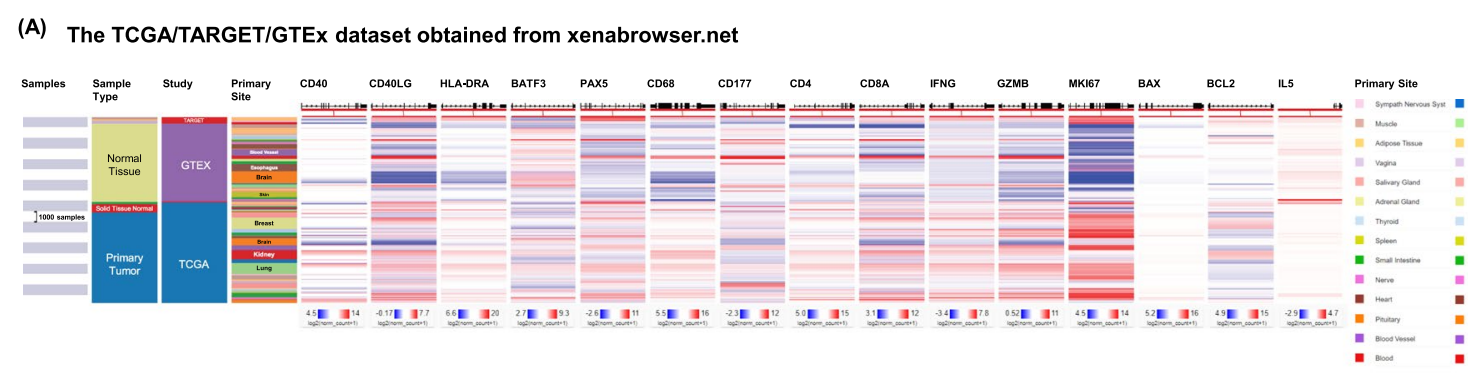

(B) Spearman's rank correlation coefficient Normal tissue $(n=8,152)$

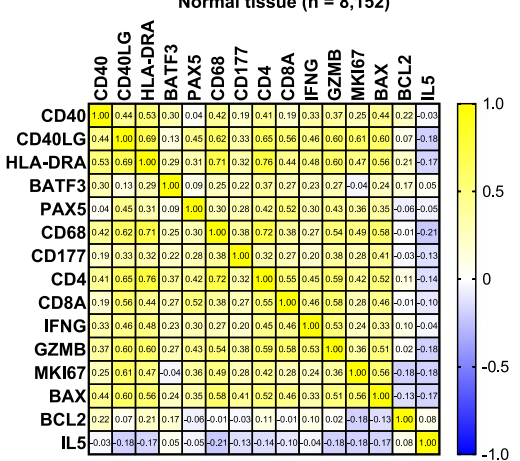

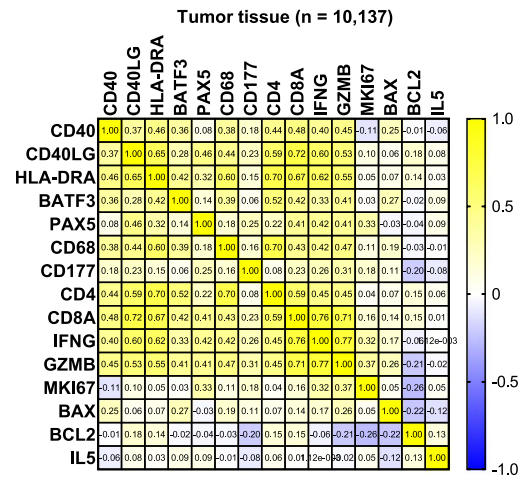

(C)

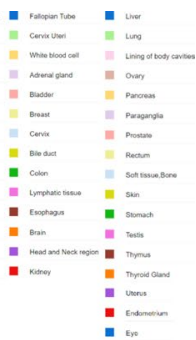
Kaplan Meier gene expression
RNAseq - RSEM norm count

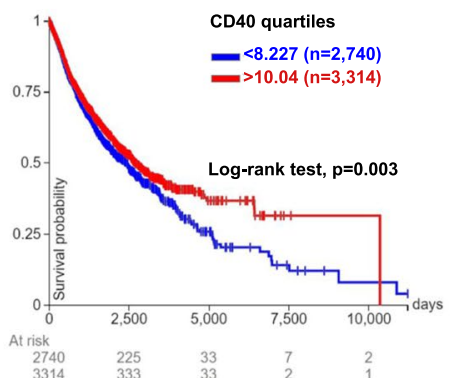

Fig. 1 CD40 expression is positively correlated with type I anti-tumor T-cell responses and better survival. A Heat map of indicated gene expression across normal and tumor tissues in the TCGA/TARGET/GTEx database obtained from the UCSC Xena web-based tool. B Correlation matrix with Spearman's rank correlation coefficients of RNA-Seq normalized count of indicated genes. C Kaplan Meier survival plot with log-rank test of pan-cancer samples stratified by CD40 quartiles of RNA-Seq normalized count

moderately co-expressed with CD40LG in the normal tissues $(r=0.44)$ and in the tumor microenvironment (TME, $r=0.37$ ). In normal tissues, $C D 40$ is positively associated with type-I responses (IFNG, $r=0.33$ and $G Z M B, r=0.37)$ and response to antigen-presentation (HLA-DRA, $r=0.53)$, $\mathrm{T}$ helper (Th) cells $(C D 4$, $r=0.41)$, but not cytotoxic T cells $(C D 8 A, r=0.19)$. Additionally, the closest association of cell proliferation (MKI67) is with CD4OLG $(r=0.61)$ and proapoptotic marker $B A X(r=0.56)$ [5], suggesting that the CD40/CD40LG axis is important in the process of immune cell turnover and homeostasis under normal conditions. We found that CD68 (macrophage), but not $C D 177$ (neutrophil), is positively correlated with $C D 40$ in both normal $(r=0.42)$ and tumor $(r=0.38)$ tissues. In addition, $C D 68$ (macrophage) is highly correlated to antigen-presentation responses (HLA-DRA, $r=0.71$ and 0.60 in normal and tumor tissues, respectively) and moderately correlated to GZMB ( $r=0.54$ and 0.47 in normal and tumor tissues, respectively). In the TME, we found that CD40 expression is moderately correlated with type-I T-cell and APC responses as reflected by significant coexpression with $H L A$ DRA $(r=0.46), C D 4(r=0.44), C D 8 A(r=0.48)$, IFNG $(r=0.40)$ and GZMB $(r=0.45)$. Furthermore, CD40 is moderately correlated with BATF3 (dendritic cells) in normal $(r=0.30)$ and tumor $(r=0.36)$ tissues. In contrast, CD4OLG is moderately correlated with PAX5 (B cells, $r=0.45$ and 0.46 in normal and tumor tissues, respectively), but not $B A T F 3$ (dendritic cells). In tumors, pan-APC marker HLA-DRA (MHC molecule), BATF3 (dendritic cells), PAX5 (B cells) and CD68 (macrophages) exhibited a positive correlation $(r>0.3)$ with CD8A, IFNG and GZMB. Together, these results suggested that while the CD40/CD40LG signal may be provided by APCs in a context-dependent manner, it is associated with beneficial T-cell-mediated cytotoxic effects (CD8A, IFNG and GZMB). The observed association between $C D 40$ expression and the type-I anti-tumor T-cell response could partially result from $\mathrm{CD} 40^{+} \mathrm{CD}^{+} \mathrm{T}$ cells in the TME, since $\mathrm{CD} 40$ activation is fundamental for their memory generation [6]. There is no correlation between CD40/C4OLG and the markers of cell death $(B A X)$ or cell survival (BCL2) in the TME. In addition, the CD40/CD40LG axis exhibited no correlation with type-II pro-tumor responses (IL5) in either normal or cancer tissues. Consistently, high $C D 40$ expression in tumors is associated with a better survival of cancer patients (Fig. 1C, log-rank test, $p=0.003)$. 


\section{CD40 regulation and connection to RAS/RAF/ PI3K-targeted and immune therapies}

After identifying the anti-tumoral role of CD40/CD40LG in pan-cancer analysis, we next explored whether any cancer type(s) may alter CD40 in tumorigenesis (Fig. 2A). Among the 23 cancer types screened, CD40 downregulation was observed in 11 malignancies compared to corresponding normal tissues, including brain, breast, lung, colon, ovary, esophagus, skin, prostate, uterus, endometrium, and blood cancers. In contrast, five malignancies (thyroid, testis, head and neck, adrenal, and kidney) exhibited an increased CD40 expression in the established tumors. Given that melanocytes comprise a minor population $(<10 \%)$ in normal skin [7], the direct comparison between normal skin and melanoma tissues may be biased by the predominant keratinocyte composition in the epidermal basal layer. Thus, we further validated the $C D 40$ expression in nevus samples and compared it to expression in melanoma tissues using the NCBI-GSE112509 bulk RNA-Seq dataset (Fig. 2B) [8]. There is no significant alteration of $C D 40$ expression among $N R A S^{w t} / B R A F^{w t}$ nevi, $N R A S^{w t} / B R A F^{m u t}$ nevi and $N R A S^{w t} / B R A F^{w t}$ melanoma tissues. However, we observed heterogeneity of $C D 40$ expression among the melanoma tissues bearing oncogenic NRAS and BRAF mutations. A reduced $C D 40$ expression was observed in $N R A S^{w t} / B R A F^{m u t}$ melanoma compared to $N R A S^{w t} /$ $B R A F^{\text {mut }}$ nevus tissues. Furthermore, NRAS ${ }^{\text {mut }}$ melanoma exhibited a reduced $C D 40$ expression compared to either $N R A S^{w t} / B R A F^{w t}$ nevi, $N R A S^{w t} / B R A F^{m u t}$ nevi or $N R A S^{w t} / B R A F^{w t}$ melanoma tissues. Consistently, $R A S$ $(N / H / K)$ mutation alone $(p=0.058)$, or in combination with NF1 mutation $(p=0.038)$, was associated with a reduced $C D 40$ expression in TCGA-melanoma (Firehose Legacy database, $n=479$ ) (Fig. $2 \mathrm{C}$ ). These results match well with the observation that RAS/RAF/PI3K pathway inhibition increased CD40 expression in melanoma cells [2].

We next explored the pathway network of human CD40 based on the Pathway Interaction Database curated by NCI/Nature (http://www.ndexbio.org/). The CD40/CD40LG axis may lead to the activation of MAPK, PI3K/AKT, NF- $\mathrm{kB}$ and JAK3/STAT5 pathways (Fig. 2D, clustering coefficient $=0.379$ ), which are known RAS-downstream signaling effector pathways [9]. Thus, when therapies that inhibit the RAS/RAF/PI3K pathway are utilized, CD40 can be upregulated, allowing CD40mediated compensatory activation of the RAS-downstream signaling activity. Also, human melanoma cells with increased CD40 at either copy number or mRNA

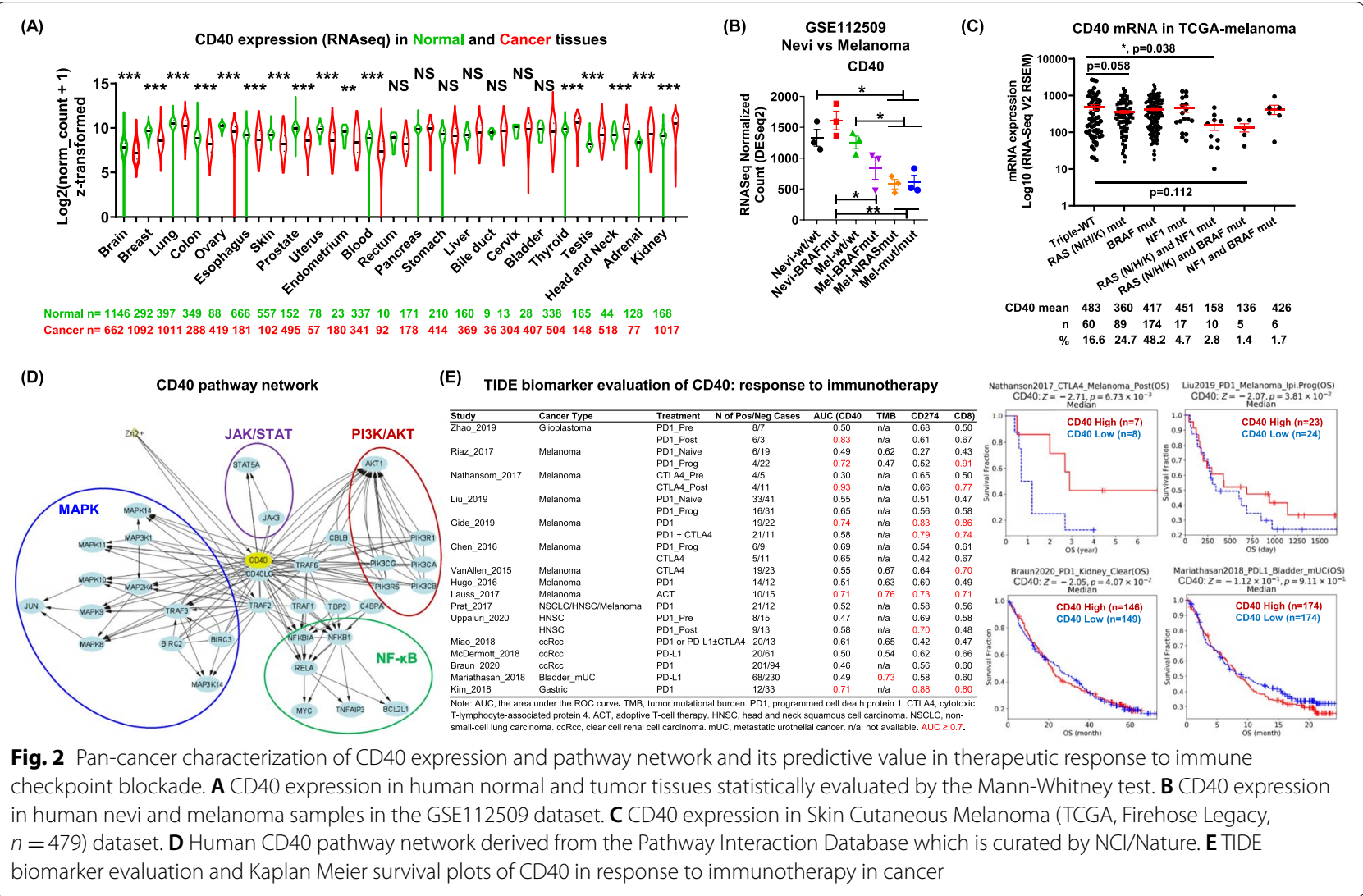


expression levels are significantly more sensitive to the RAF inhibitor treatments [2]. The compensatory regulation of CD40 signaling mediated by the RAS/RAF/PI3Kpathway might be utilized in tumors with low intrinsic CD40 levels, such as NRAS ${ }^{\text {mut }}$ melanoma. Indeed, MEK inhibition (MEKi) increased survival of NRAS mut melanoma patients [10]. The results from the NEMO trial comparing the efficacy of a MEK inhibitor (MEK162) versus dacarbazine in unresectable or metastatic NRASmut melanoma revealed that the MEK162 treatment group had 2.8-month PFS compared to the chemotherapy group with a 1.5-month survival. However, when the analysis was confined to patients who had received prior immunotherapy, the MEK162 PFS was 5.5 months compared to 1.6 months for the chemotherapy treatment group. These data further support the notion that NRASmut melanoma patients who received first-line immune checkpoint blockade therapy might benefit from MEKi therapy $[11,12]$.

Recent advances in ICB therapy, such as targeting programmed cell death protein 1 (PD-1)/PD-ligand 1 (PD-L1) and/or cytotoxic T-lymphocyte-associated protein 4 (CTLA-4), illustrate powerful enhancement of the patient's anti-tumor immune responses [13]. Currently, ICB is a first-line therapy for several cancer types, including unresectable metastatic melanoma. To evaluate whether there might be a predictive value of CD40 for immunotherapy, we utilized the Tumor Immune Dysfunction and Exclusion (TIDE) framework (tide.dfci. harvard.edu), which is a computational framework developed to evaluate potential of tumor immune escape based on the gene expression profiles of cancer samples. The TIDE framework data base covers glioblastoma $(n=24)$, head and neck squamous cell carcinoma (HNSC, $n>45$ ), non-small-cell lung carcinoma (NSCLC, $n<33$ ), kidney cancer $(n=408)$, bladder cancer $(n=298)$, gastric cancer $(n=45)$ and 12 melanoma cohorts $(n>426)$. We analyzed the area under the ROC curve (AUC) for CD40 expression in comparison with existing biomarker signatures, including tumor mutational burden (TMB), CD274 (PD-L1) and CD8 [14], as a tool for predicting response to immunotherapy (Fig. 2E). The prediction performances of CD40 are comparable to CD8 in $\geq 50 \%$ melanoma cohorts and one gastric cancer cohort, where both biomarkers exhibited an AUC $>0.7$, predicting a strong likelihood of positive response to immunotherapy. Notably, CD40 is the only biomarker that achieved a good performance in predicting ICB response in glioblastoma $(\mathrm{AUC}=0.83)$. TMB $(\mathrm{AUC}=0.73)$ and $\mathrm{CD} 274$ $(\mathrm{AUC}=0.7)$ are predictive for ICB response in one bladder cancer cohort and one HNSC cohort, respectively. None of these biomarkers exhibited good performance in NSCLC or kidney cancer. All the biomarkers in the pre-treatment or treatment-naive biopsies from the cancers evaluated here (Fig. 2E) had a prediction score lower than 0.7 , suggesting that these biomarkers may be more predictive of treatment-induced responses. In general, the predictive ability of these transcriptomic biomarkers to immunotherapy response is still currently limited. Notably, high CD40 is associated with better overall survival (OS) in two melanoma cohorts of patients treated with $\alpha$-CTLA4 $(n=15, p=0.0067)$ or $\alpha$-PD1 $(n=47$, $p=0.0381)$. Nevertheless, there is either no correlation or a negative correlation of CD40 expression level and OS of patients who received $\alpha$-PD-L1 in bladder cancer ( $n=348, p=0.9110$ ) or patients who received $\alpha$-PD1 in kidney cancer $(n=295, p=0.0407)$. The cancer-typespecific predictive performance of CD40 in ICB response suggests additional features of the TME may be involved in bladder and kidney cancers.

There are limitations to our study. For instance, the analysis of mRNA markers and immune profiling in tumors are derived from selected databases and should be interpreted with caution; future functional characterization and validation on the role of CD40 in immune cells versus non-immune cells will be important to determine whether CD40 is a driver or bystander in the specific experimental settings [15]. In the survival analysis using TIDE biomarker evaluation on CD40, results were dichotomized at cohort-specific median of z-scores, which warrants future trials in a larger cohort to validate the role of CD40 in treatment responses to patient's survival and additional clinical characteristics, e.g., metastatic status. In general, the effect of CD40 on tumorigenesis and response to therapy (sensitivity and toxicity) may largely depend on the context of the tumor type and cancer stage, cytokine availability, ligand/receptor distribution, and crosstalk among different stimuli, cell types, and signaling pathways.

\section{Conclusion}

We have shown a close correlation of the CD40/CD40LG axis with type-I anti-tumor T-cell responses in cancer. The prevalence of reduced CD40 expression in tumor versus normal tissue was identified in many cancer types, including melanoma. $R A S(N / H / K)$ mutation correlates with low CD40 expression in melanoma. Moreover, CD40 is a potential predictive biomarker of response to ICB therapy for some tumor types (melanoma, gastric cancer, and glioblastoma).

\footnotetext{
Abbreviations

APC: Antigen presenting cells; CD40LG: CD40 ligand; ICD: Immunogenic cell death; MHC: Major histocompatibility complex; ICB: Immune checkpoint blockade; TME: Tumor microenvironment; Th cells: Thelper cells; PD-1: Programmed cell death protein 1; PD-L1: PD-1 ligand 1; CTLA-4: Cytotoxic T-lymphocyte-associated protein 4; TIDE: Tumor immune dysfunction and
} 
exclusion; HNSC: Head and neck squamous cell carcinoma; NSCLC: Non-smallcell lung carcinoma; AUC: Area under the ROC curve; TMB: Tumor mutational burden; OS: Overall survival;TCGA: The cancer genome atlas.

\section{Supplementary Information}

The online version contains supplementary material available at https://doi. org/10.1186/s12943-021-01442-3.

Additional file 1. Supplementary Methods.

\section{Acknowledgements}

N/A

\section{Authors' contributions}

$C Y$ and $A R$ designed the research approach; $C Y$ performed the experiments and analyzed data; $C Y$ made a draft of the paper; $C Y$ and AR revised and edited the paper. The authors read and approved the final manuscript.

\section{Funding}

This work was supported by a VA Merit award (I01BX002301) and a VA SRCS award to AR and by R01-CA116021 (AR).

\section{Availability of data and materials}

Supplementary Methods can be accessed online.

\section{Declarations}

Ethics approval and consent to participate N/A

\section{Consent for publication}

All authors have agreed to publish this manuscript.

\section{Competing interests}

There are no potential conflicts of interest to disclose

\section{Author details}

${ }^{1}$ Tennessee Valley Healthcare System, Department of Veterans Affairs, Nashville, TN, USA. ${ }^{2}$ Department of Pharmacology, Vanderbilt University School of Medicine, 432 PRB, 2220 Pierce Ave, Nashville, TN 37232, USA

Received: 20 July 2021 Accepted: 4 October 2021

Published online: 10 November 2021

\section{References}

1. Chand Dakal T, Dhabhai B, Agarwal D, Gupta R, Nagda G, Meena AR, et al. Mechanistic basis of co-stimulatory CD40-CD40L ligation mediated regulation of immune responses in cancer and autoimmune disorders. Immunobiology. 2020;225:151899.

2. Yan C, Saleh N, Yang J, Nebhan CA, Vilgelm AE, Reddy EP, et al. Novel induction of CD40 expression by tumor cells with RAS/RAF/PI3K pathway inhibition augments response to checkpoint blockade. Mol Cancer. 2021;20:85

3. Pirozzi G, Lombari V, Zanzi D, Ionna F, Lombardi ML, Errico S, et al. CD40 expressed on human melanoma cells mediates T cell co-stimulation and tumor cell growth. Int Immunol. 2000;12:787-95.

4. von Leoprechting A, van der Bruggen P, Pahl HL, Aruffo A, Simon JC. Stimulation of CD40 on immunogenic human malignant melanomas augments their cytotoxic T lymphocyte-mediated lysis and induces apoptosis. Cancer Res. 1999;59:1287-94.

5. Rathmell JC, Lindsten T, Zong WX, Cinalli RM, Thompson CB. Deficiency in Bak and Bax perturbs thymic selection and lymphoid homeostasis. Nat Immunol. 2002;3:932-9.

6. Bourgeois C, Rocha B, Tanchot C. A role for CD40 expression on CD8+ T cells in the generation of CD8+ T cell memory. Science. 2002;297:2060-3.

7. Cichorek M, Wachulska M, Stasiewicz A, Tyminska A. Skin melanocytes: biology and development. Postepy Dermatol Alergol. 2013:30:30-41.

8. Kunz M, Loffler-Wirth H, Dannemann M, Willscher E, Doose G, Kelso , et al. RNA-seq analysis identifies different transcriptomic types and developmental trajectories of primary melanomas. Oncogene. 2018:37:6136-51

9. Gimple RC, Wang X. RAS: striking at the core of the oncogenic circuitry. Front Oncol. 2019;9:965.

10. Kirchberger MC, Ugurel S, Mangana J, Heppt MV, Eigentler TK, Berking C, et al. MEK inhibition may increase survival of NRAS-mutated melanoma patients treated with checkpoint blockade: results of a retrospective multicentre analysis of 364 patients. Eur J Cancer. 2018;98:10-6.

11. Dummer R, Schadendorf D, Ascierto PA, Arance A, Dutriaux C, Di Giacomo AM, et al. Binimetinib versus dacarbazine in patients with advanced NRAS-mutant melanoma (NEMO): a multicentre, open-label, randomised, phase 3 trial. Lancet Oncol. 2017;18:435-45.

12. Bersanelli M, Leonetti $A$, Buti S, Michiara M. "Finding NEMO" in NRASmutant melanoma: a step towards a sequential strategy? Lancet Oncol. 2017;18:e298.

13. Tang T, Huang X, Zhang G, Hong Z, Bai X, Liang T. Advantages of targeting the tumor immune microenvironment over blocking immune checkpoint in cancer immunotherapy. Signal Transduct Target Ther. 2021;6:72.

14. Litchfield K, Reading JL, Puttick C, Thakkar K, Abbosh C, Bentham R, et al. Meta-analysis of tumor- and T cell-intrinsic mechanisms of sensitization to checkpoint inhibition. Cell. 2021;184:596-614 e514.

15. Djureinovic D, Wang M, Kluger HM. Agonistic CD40 antibodies in cancer treatment. Cancers (Basel). 2021;13:1302.

\section{Publisher's Note}

Springer Nature remains neutral with regard to jurisdictional claims in published maps and institutional affiliations.

Ready to submit your research? Choose BMC and benefit from:

- fast, convenient online submission

- thorough peer review by experienced researchers in your field

- rapid publication on acceptance

- support for research data, including large and complex data types

- gold Open Access which fosters wider collaboration and increased citations

- maximum visibility for your research: over 100M website views per year

At BMC, research is always in progress.

Learn more biomedcentral.com/submissions 